\title{
Performance Analysis of Coherent Atmospheric Optical Communications with Soft-decision Forward Error Correction
}

\author{
Jinjing Tao, Jinnan Zhang, Yangan Zhang, Yongqing Huang, Xueguang Yuan, Yupeng Li \\ State Key Laboratory of Information Photonics and Optical Communications \\ BUPT \\ Beijing, China \\ E-mail: taojinjing@bupt.edu.cn
}

\begin{abstract}
Performance of coherent atmospheric optical communication system with heterodyne detection and LDPC codes was evaluated over atmospheric channel attenuations of which are about 20-30 dB/ $/ \mathrm{km}$. To reduce bit error and enhance the system performance LDPC code was implemented in system. Combining coherent detection and LDPC codes could reduce the received power requirement $\sim 4 \mathrm{dBm}$ at the $B E R$ of $10^{-9}$
\end{abstract}

Keywords-atmospheric optical communication; coherent detection; forward error correction (FEC); low-density paritycheck (LDPC) codes

\section{INTRODUCTION}

Atmospheric optical communication system could be a promising option for applications requiring low cost, high bandwidth and enhanced security. However unfavorable weather conditions such as fog, rain and snow make it difficult to ensure the quality of the communication using atmospheric optical communication system.

Soft-decision forward error correction (FEC) is becoming practical in coherent receivers [1]. Low-density parity-check (LDPC) codes, first proposed by Gallager in the 1960's [2], could help improve the system performance [3][4][5] and is a good candidate for a strong soft-decision based FEC.

Rain may cause channel attenuations up to $20-30 \mathrm{~dB} / \mathrm{km}$ at a rain rate of $150 \mathrm{~mm} / \mathrm{h} \mathrm{[6].} \mathrm{In} \mathrm{this} \mathrm{paper,} \mathrm{the} \mathrm{system}$ performance was evaluated in such weather condition. The coherent atmospheric optical communication system proposed and demonstrated implemented heterodyne detection in receiver which helped improving the sensitivity and optimizing system performance. A rate-5/6 (redundancy $20 \%$ ) $\pi$-rotation LDPC code [7][8] was adopted in system because of its less encoding process complexity and easy hardware implementation. The bit-error ratio (BER) performance of LDPC-coded coherent atmospheric optical communication system was studied and compared with the system which does not employing LDPC codes. The system sensitivity was increased according to the comparison results. The received power required at the receiver decreased to around $4 \mathrm{dBm}$ at the BER of $10^{-9}$.

\section{LOW-DENSITY PARITY-CHECK CODES}

Low-density parity-check (LDPC) codes are linear block codes whose parity-check matrix $\mathrm{H}$ is sparse which means it has a low density of ones. In brief, there are two sets of methods to construct LDPC codes. One set of methods is based on random constructions, while the second based on algebraic constructions [9]. LDPC codes produced by random construction have the near Shannon limit error performance. However it is quite difficult to implement the randomly constructed LDPC codes in hardware because of the encoding and decoding complexity introduced by random construction. The algebraically constructed LDPC codes are more suitable for hardware realization. The $\pi$-rotation LDPC codes are one of algebraically constructed LDPC codes introduced in as a class of quasi-regular LDPC codes. Its construction is based on the $\pi$-rotation structure using a single permutation matrix to define the code. Since the encoding process of $\pi$-rotation LDPC code is of less complexity and implemented easily in hardware, the $\pi$ rotation LDPC code was the soft-decision FEC used in the proposed transmission system.

\section{SySTEM SETUP}

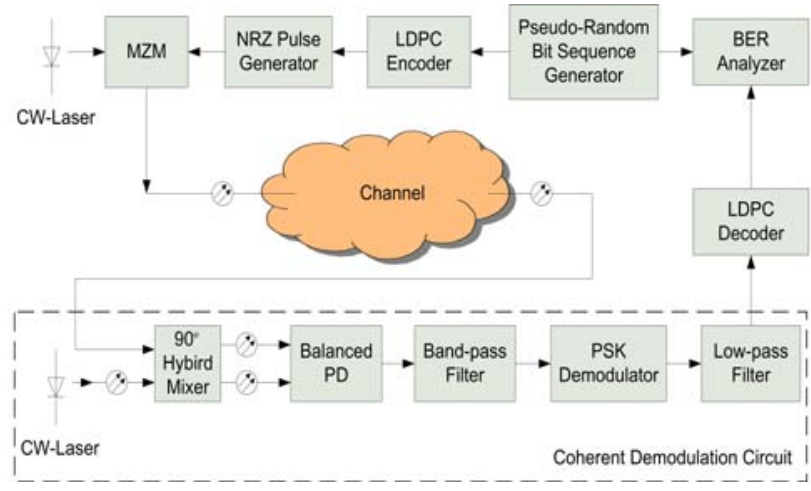

Figure 1. Experimental bed setup.

To evaluate the performance of the LDPC-coded coherent atmospheric optical communication using heterodyne detection, a transmission system was set up as illustrated in Fig. 1. The transmitter consisted of a pseudorandom bit sequence (PRBS) generator, a LDPC encoder, a CW laser and one chirp-free Mach-Zehnder Modulator (MZM). The laser employed as light source in the system was a tunable $\mathrm{CW}$ laser with tuning rage from 1520 to 1600 $\mathrm{nm}$ with $50 \mathrm{kHz}$ linewidth. The PRBS generator generated one $2^{11}-1$ pseudo-random binary sequence encoded in the LDPC encoder using $\pi$-rotation LDPC codes of code rate $\mathrm{r}=5 / 6$. A $10 \mathrm{Gbit} / \mathrm{s} \mathrm{NRZ}$ electrical pulse train based on the 
encoded bit stream was produced by the NRZ pulse generator. Light from the laser which had set its centre frequency at $193.4 \mathrm{THz}$ was modulated in chirp-free MachZehnder Modulator (MZM) driven by the $10 \mathrm{Gbit} / \mathrm{s}$ NRZ signal. NRZ-DPSK signal was generated. The optical spectrum for the light source is shown in Fig. 2.

A $0.2 \mathrm{~nm}$ narrow-band optical filter was used to depress the background noise in the received optical signal transmitted through the atmospheric channel. Then the received optical signal was mixed with a local oscillator in the $90^{\circ}$ optical hybrid mixer. The centre frequency of the local oscillator was set at $193.45 \mathrm{THz}$ and the linewidth of the local oscillator was $50 \mathrm{kHz}$ also. The heterodyne detection was achieved. The output from the mixer was fed to the balanced detector circuit. The PSK demodulation was accomplished by PSK demodulator. Low-pass filter helped suppress the noise. LDPC decoder first sampled and quantized the received NRZ electrical signal demodulated from the former circuit. The LDPC decoder output a binary stream of which the length was the same as the binary sequence produced by the PRBS generator. BER was calculated by the BER analyzer. Eye pattern could also be observed before LDPC decoding. In the proposed system the uniformly most powerful (UMP) BP-based algorithm [10] was implemented in LDPC decoder.

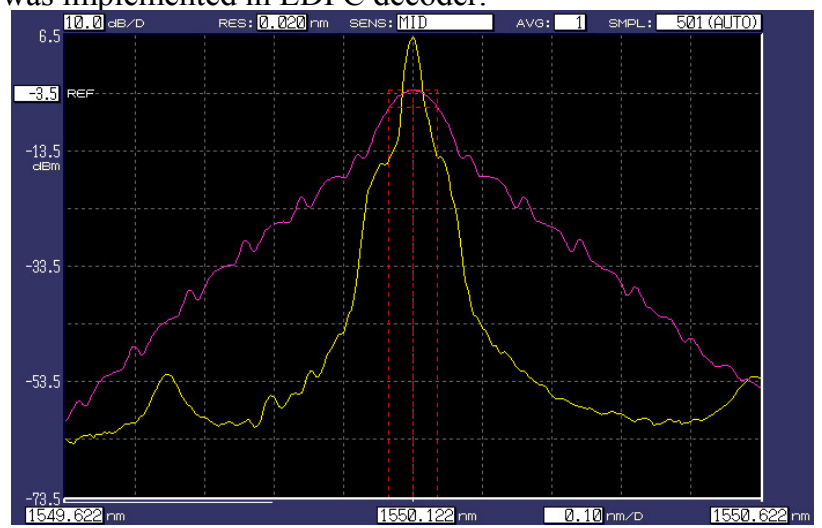

Figure 2. Measured optical spectrum for light source (yellow) and transmitted signal (pink)

\section{NUMERICAL RESUlTS}

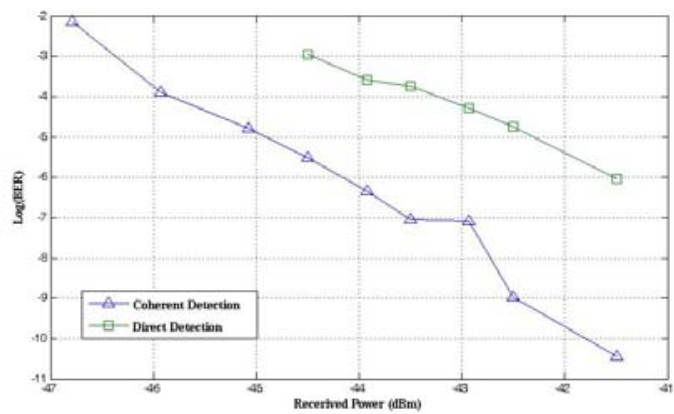

Figure 3. BER curves for coherent detection and direct detection.
Limited by the experimental conditions, we assessed the performance of the proposed system by exploiting simulation platform. We simulated the performance of the coherent atmospheric optical transmission system with different atmospheric channel attenuation characteristics. The transmission range was set to $1 \mathrm{~km}$. The aperture diameter of the transmitter telescope was $50 \mathrm{~mm}$ and the aperture diameter of the receiver telescope was $200 \mathrm{~mm}$. The BER curves in Fig. 3 illustrate the comparison of the LDPC-coded atmospheric optical transmission systems with coherent heterodyne detection and direct detection. When BER equaled to $10^{-3}$, the received power of the system was about $-44.5 \mathrm{dBm}$ using direct detection and $-46.6 \mathrm{dBm}$ using coherent heterodyne detection. The received power decreased about $2 \mathrm{dBm}$ at the BER of $10^{-3}$. The receiving sensitivity was improved.

Finally degradation in performance of the atmospheric optical transmission systems caused by the atmospheric attenuations due to weather effects was investigated. The atmospheric attenuation factor was adjusted range from 20 to $30 \mathrm{~dB} / \mathrm{km}$. It helped us analyze the impact of the atmospheric attenuation on system performance. All other atmospheric channel parameters were set to be the same as before. To calculate the BER of the transmission system the signal input into the LDPC decoder was first sampled and quantized. Then the sampled and quantized signal was used to get a decision train with hard decision. We computed the BER which was represented by the blue curve in Fig. 4 by comparing the decision train got from hard decision with the binary sequence generated by the PRBS generator. The green curve in Fig. 4 was obtained by comparing the decoded bit stream output from the LDPC decoder with the binary sequence generated by the PRBS generator. The decision train, the decoded bit stream and the binary sequence had the same length. Fig. 4 illustrated that LDPC codes would help correct error bit and improve the BER performance dramatically. When the received power was beyond -47.4 $\mathrm{dBm}$ the system could achieve the BER of $10^{-12}$ when LDPC decoding was accomplished. At the BER of $10^{-9}$ the received power of the system decreased about $4 \mathrm{dBm}$.

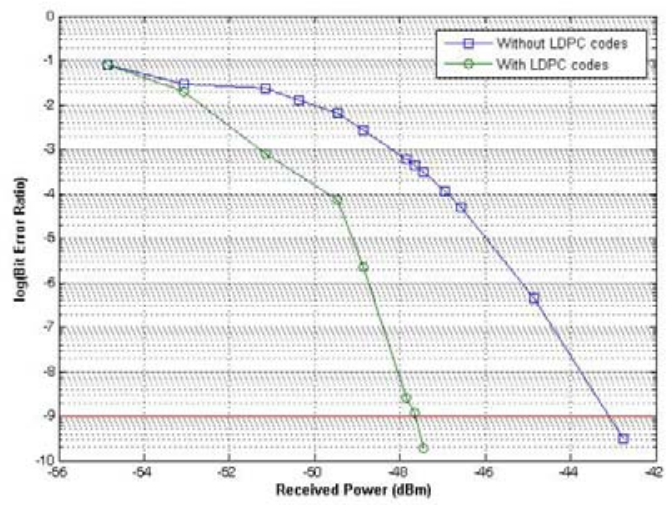

Figure 4. The BER performance for the system with and without LDPC decoding. 


\section{CONCLUSION}

We studied the performance of the coherent atmospheric optical communication system over the atmospheric channel of which the attenuation factor was at $20-30 \mathrm{~dB} / \mathrm{km}$. The LDPC-coded coherent atmospheric optical communication system that we proposed worked efficiently and steadily. It was demonstrated that coherent heterodyne detection could, to some extent, improve the receiving sensitivity of the system. The received power of the coherent atmospheric optical communication system could decrease $\sim 2 \mathrm{dBm}$ at the BER of $10^{-3}$ compared with the direct detection atmospheric optical communication system. Moreover, the LDPC codes employed as a soft-decision FEC in transmission system would help reduce the bit error to a certain degree. Exploiting coherent heterodyne detection with soft-decision FEC in atmospheric optical communication system could lower the received power and bit-error ratio. The required received power for the system reduced $\sim 4 \mathrm{dBm}$ at the BER of $10^{-9}$. The performance of the system was quite acceptable.

\section{ACKNOWLEDGMENT}

This work is supported by the fundamental research funds for the Central Universities (2009RC0401), the fund of Young Scholar Innovation Project (2011PTB-00-31), the fund of Young Scholar Innovation Project (2012RC0407) and the fund of Young Scholar Innovation Project (2012RC0406).

\section{REFERENCES}

[1] T. Mizuochi, T. Sugihara, Y. Miyata, K. Kubo, K. Onohara, S. Hirano, H. Yoshida, T. Yoshida, and T. Ichikawa "Evolution and Status of Forward Error Correction," OFC 2012, OTu2A.6, 2012.

[2] R. G. Gallager, "Low-Density Parity-Check Codes," IRE Transactions on Information Theory, vol. 8, pp. 21-28, January 1962.

[3] Ivan B. Djordjevic, Murat Arabaci and Lyubomir L. Minkov, "Next Generation FEC for High-Capacity Communication in Optical Transport Networks," J. Lightw. Technol., vol. 27, no. 16, On page(s).3518-3530, August 2009.

[4] Jiaojiao Fu, Murat Arabaci, Ivan B. Djordjevic, Yequn Zhang, Lei Xu and Ting Wang, "First Experimental Demonstration of Nonbinary LDPC-Coded Modulation Suitable for High-Speed Optical Communications," OFC 2011, OWF4, 2011.

[5] Cai, Jin-Xing, Batshon, Hussam G., Zhang, Hongbin, Davidson, Carl, Sun, Yu, Mazurczyk, Matt, Foursa, Dmitri, Pilipetskii, Alexei, Mohs, Georg and Bergano, Neal, "25 Tb/s Transmission over $5,530 \mathrm{~km}$ Using 16QAM at $5.2 \mathrm{bits} / \mathrm{s} / \mathrm{Hz}$ Spectral Efficiency," ECOC 2012, Mo.1.C.1, 2012.

[6] L. Castanet, "Influence of the variability of the Propagation channel on mobile, fixed multimedia and optical satellite communications," JA2410 SatNEx e-Book, Shaker Verlag 2007.

[7] Rich Echard and Shih-Chun Chang, "The $\pi$-Rotation Low-Density Parity Check Codes," Global Telecommunications Conference, 2001, On page(s): 980-984.

[8] Rich Echard and Shih-Chun Chang, "Deterministic $\pi$-rotation lowdensity parity-check codes," Electronics Letters, vol. 38, No. 10, 2002.

[9] Yige Wang, Jonathan S. Yedidia and Stark C. Draper, "Construction of High-Girth QC-LDPC Codes," Turbo Codes and Related Topics, 2008 5th International Symposium on, On page(s): 180-185.

[10] Jinghu Chen and Marc P.C. Fossorier, "Decoding Low-Density Parity Check Codes with Normalized APP-Based Algorithm," Global Telecommunications Conference, 2001, On page(s): 1026-1030. 